\title{
Subclinical left ventricular systolic dysfunction in patients with naive acromegaly - assessment with two-dimensional speckle-tracking echocardiography: retrospective study
}

\author{
Agata Popielarz-Grygalewicz', Maria Stelmachowska-Banaś2, Jakub S. Gąsiorr, 3, Paweł Grygalewicz', \\ Magdalena Czubalska ${ }^{1}$, Wojciech Zgliczyński ${ }^{2}$, Marek Dąbrowski', Wacław Kochman ${ }^{1}$ \\ ${ }^{1}$ Clinical Department of Cardiology of National Institute of Cardiology, Bielanski Hospital, Warsaw, Poland \\ ${ }^{2}$ Department of Endocrinology of the Centre of Postgraduate Medical Education, Warsaw, Poland \\ ${ }^{3}$ Faculty of Health Sciences and Physical Education, Kazimierz Pulaski University of Technology and Humanities, Radom, Poland \\ ${ }^{4}$ Independent Public Healthcare Centres (Bemowo-Wlochy), Warsaw, Poland
}

\begin{abstract}
Introduction: The aim of the study was to evaluate global longitudinal strain (GLS) in patients with naive acromegaly with normal left ventricular (LV) ejection fraction (EF).

Material and methods: Forty-three consecutive patients with naive acromegaly with normal LV systolic function as measured by EF, examined from 2008 to 2016, and 52 patients of a control group matched for age and sex underwent two-dimensional speckle-tracking echocardiography to assess GLS.

Results: The median GLS was significantly lower in the acromegaly group than in the control group (in \%, -16.6 vs. $-20.7 ; p<0.01$ ). The majority of acromegalic patients $(n=26 ; 60.5 \%)$ had abnormal GLS. Patients with impairment in GLS had a longer median duration of acromegaly symptoms (in years, $10.0 \mathrm{vs} .5 .0 ; \mathrm{p}<0.05$ ) and greater LV thickness (posterior wall in mm, $12.5 \mathrm{vs} .12 .0 ; \mathrm{p}<0.05$ ) compared to those with normal GLS. Patients with abnormal GLS had higher IGF-1 concentration, but without statistical significance. Diabetes mellitus and arterial hypertension, which are more common in acromegaly, were not significant determinants of abnormal GLS. The mean left ventricular mass index (LVMI) was increased in the acromegaly group compared to controls (in $\mathrm{g} / \mathrm{m}^{2}, 136$ vs. 97; $\left.\mathrm{p}<0.01\right)$. There was a significant negative correlation between LVMI and GLS $(\mathrm{R}=-0.47 ; \mathrm{p}<0.01)$.

Conclusions: Naive acromegalic patients presented abnormal GLS, which indicates subclinical systolic dysfunction in these patients. It has not been proven that arterial hypertension and diabetes mellitus are significant determinants of abnormal GLS. (Endokrynol Pol 2020; 71 (3): 227-234)
\end{abstract}

Key words: acromegaly; global longitudinal strain; left ventricular systolic dysfunction; speckle tracking echocardiography; left ventricular ejection fraction; insulin growth factor-1; growth hormone

\section{Introduction}

Acromegaly is a rare endocrine disease characterised by increased growth hormone (GH) and insulin-like growth factor 1 (IGF-1) levels. It is usually caused by GH-secreting pituitary adenoma and rarely by neuroendocrine tumours producing growth hormone-releasing hormones [1-2].

Acromegaly is a systemic disease that affects different organs, including the cardiovascular system, and leads to many comorbidities [3].

The main marker in the assessment of heart involvement in the course of various diseases is systolic function as measured by ejection fraction (EF). This parameter, evaluated by end-diastolic and end-systolic volumes according to the biplane Simpson method, remains a crucial determinant of the risk stratification of heart failure patients, according to current guidelines [4].

However, the left ventricular ejection fraction (LVEF) may not truly represent left ventricular (LV) systolic function in specific cardiac syndromes, such as asymptomatic severe valve diseases, as well as in many other systemic, metabolic, and endocrine disorders [5-7].

Two-dimensional speckle-tracking echocardiography (2D-STE) is a new technique that allows the evaluation of longitudinal, radial, and circumferential deformation and offers a more sensitive assessment of myocardial contractility [5]. It is based on the tracking of characteristic speckle patterns that are created by the interference of ultrasound beams in all segments of myocardium $[8,9]$. The strain is the percentage change in the myocardial fibre length between end-diastole and end-systole, and, depending on the type of fibre, this change is local short- 
ening, thickening, or lengthening. Finally, the average value of the strain of all segments is obtained [10]. From all these measurements the global longitudinal strain (GLS) is calculated, which is the best measure of global LV systolic function and provides relevant evidence on the diagnostic and prognostic implications $[5,11,12]$.

There are many clinical scenarios in which subtle LV systolic dysfunction is present despite normal EF and can be characterised with GLS $[6,7,13]$.

It seems that the use of new techniques to assess systolic function in acromegalic patients may shed new light on the controversial diagnosis of acromegalic cardiomyopathy.

The aim of this study was to determine if patients with naive acromegaly, with preserved LVEF, present abnormal GLS in comparison with a matched control group.

\section{Material and methods}

\section{Study population}

A total of 140 consecutive patients diagnosed with acromegaly at the Department of Endocrinology of the Centre of Postgraduate Medical Education, Warsaw, Poland, from 2008 to 2016, underwent echocardiography at the Cardiology Clinic of Physiotherapy Division of the Second Faculty of Medicine of the Medical University of Warsaw as a routine diagnostic procedure. The patients were at various stages of the disease. Out of this group, we included 43 adult patients with naive acromegaly and complete echocardiography examination, including GLS, for the final statistical analysis. Patients with a history of myocardial infarction or heart failure and impairment of $\mathrm{LV}$ systolic function ( $\mathrm{EF}<54 \%$ in women and $<52 \%$ in men) were excluded from the study. Patients diagnosed with adrenal insufficiency and/or secondary hypothyroidism first received proper replacement with hydrocortisone and/or L-thyroxine. Acromegaly was diagnosed on the basis of the following:

- typical acromegalic features. In most cases, old photos of the patient (5-20 years before) were used to estimate the onset of the disease $[1,3]$;

- lack of GH suppression $(<1.0 \mu \mathrm{g} / \mathrm{L})$ in the oral $75 \mathrm{~g}$ glucose tolerance test;

- elevated IGF-1 level in comparison to the normal values for age and sex;

- pituitary adenoma in magnetic resonance imaging.

Arterial hypertension and glucose metabolism disorders (diabetes, prediabetes) were diagnosed according to the guidelines of the Polish Arterial Hypertension Society [14] and Polish Diabetic Society [15].

Body mass index (BMI) was calculated as body mass in kilograms divided by height in metres squared $\left[\mathrm{kg} / \mathrm{m}^{2}\right]$. Values for body surface area (BSA) were recorded from the echocardiographic examination software.

The control group consisted of 52 volunteers, of both sexes, recruited from the hospital (nurses, doctors, staff employed in administration). Initial inclusion criteria were established to match controls and patients for age, sex, BMI, and prevalence of hypertension and diabetes. Due to the relatively low number of volunteers, matching for comorbidities was impossible. The final inclusion criteria were age between 20 and 80 years and BMI between 20 and $40 \mathrm{~kg} / \mathrm{m}^{2}$.

\section{Hormone assays}

$\mathrm{GH}$ was tested using the immunochemiluminescence method with an Immulite 2000 analyser (Siemens, Los Angeles, California, USA) or Liaison XL (Dia Sorin, Italy) analyser.
IGF-1 was tested using the immunochemiluminescence method with an Immulite 2000 analyser (Siemens, Los Angeles, California, USA) or Liaison XL analyser (Dia Sorin, Italy).

All plasma samples were collected in the early morning after a 12-hour fasting period.

\section{Echocardiographic examination}

Echocardiography was performed using a Vivid 4 device (Haifa, Israel) before 2012 and Vivid 9 device (Horten, Norway) from 2012 onwards. A sector transducer with a frequency of $3.2 \mathrm{MHz}$ was used. The images were stored digitally for later offline analysis using dedicated software (EchoPac PC, workstation version 113, GE Medical Systems).

\section{Strain analysis by STE}

Global longitudinal strain is the most commonly used strain-based measure of LV global systolic function $[5,10]$. It is usually assessed by 2D-STE. GLS describes the relative length change of the LV myocardium between end-diastole and end-systole:

$$
\text { GLS }(\%)=(M L s-M L d) / M L d \times 100,
$$

- where ML is myocardial length at end-systole (MLs) and enddiastole (MLd). Because MLs is smaller than MLd, peak GLS is a negative number [10].

We used the automated function imaging (AFI) application to evaluate GLS. Automated function imaging is clinically applicable and an effective means of assessing LV function due to its short acquisition time, feasibility, accuracy, and repeatability [16].

The analysis involved ECG-gated digital images in four-, threeand two-chamber apical views, and a high temporal resolution of 50-60 frames per second was obtained to assure acoustic-marker tracking. Aortic valve closure was used for timing of end-systole - it was measured conventionally using pulsed-wave Doppler in the apical five-chamber view, and clicks of opening and closing the aortic valve were marked.

If the quality of myocardial tracking was poor, the process was repeated. Only the images with appropriate tracking in all the myocardial segments were used in the analysis.

Global longitudinal strain was the average of the value that was obtained for three apical views.

In our study, the coefficient of variation (CV) of GLS was 1.6\%.

In accordance with the guidelines, a value of GLS of $-20 \%$ or lower (i.e. more negative) was taken as normal in our study [10].

\section{Standard echocardiography analyses}

The following data were recorded:

- left ventricular diastolic dimension and myocardial thickness: interventricular septum (IVS), posterior wall (PW), and left ventricular mass (LVM). Measurements were conducted in 2D imaging. The LVM was calculated using the formula of the American Society of Echocardiography modified by Devereux [17]: LVM was indexed in accordance with BSA to obtain the left ventricular mass index (LVMI) value. Left ventricular hypertrophy was defined as an LVMI of $>115 \mathrm{~g} / \mathrm{m}^{2}$ in men and $>95 \mathrm{~g} / \mathrm{m}^{2}$ in women;

- diastolic function parameters [18]:

- E'med. $[\mathrm{cm} / \mathrm{s}]$ - early diastolic velocity of the septal mitral annulus assessed with tissue Doppler exam,

- E/E' - mitral E-wave velocity divided by mitral annular velocity,

- E/A - mitral E-wave velocity divided by mitral A-wave velocity,

- left atrial volume index (LAVI), measured using standard fourchamber projection and the curvature of the diastolic surface area of the left atrium. The value was calculated by EchoPac software,

- systolic function parameters:

- EF - ejection fraction was calculated according to the Simpson formula [10]. 
In case of imaging difficulties, the visual estimation method was used, as described in the literature [19]. Ejection fraction was assessed by two independent specialists in echocardiography. In accordance with the guidelines for the lower limit of the norm, the value of EF was 54\% in women and 52\% in men [10]. Because the EF values have no practical application when they are within normal limits and many variables affect this parameter (including hydration and heart rate), in both groups, the EF value was not recorded unless the value fell below $54 \%$ in women and below $52 \%$ in men

\section{Ethics statement}

The Bioethics Committee of the Medical University of Warsaw approved the retrospective analysis of data obtained from echocardiograms of patients with acromegaly and testing of the healthy individuals representing the control group.

\section{Statistical analysis}

Normal distribution was confirmed with the Shapiro-Wilk test before data were analysed.

Depending on the distribution of variables, appropriate tests were used for further analysis.

Student's t-test was used for comparison of average values of two variables with a normal distribution (for paired data or depending on the situation). We used nonparametric analysis when the null hypothesis of normal variable distribution was rejected. The medians were compared with the Mann-Whitney U-test. Spearman's rho correlation coefficient $(\mathrm{R})$ was used for continuous variables. The $\chi^{2}$ test was used for comparison of the basic clinical characteristics between the groups. Multivariate logistic regression analysis was used to determine which variables were predictors of GLS in patients with acromegaly. The within-subject CV was calculated to assess GLS repeatability. The CV of selected group data was computed as the standard deviation divided by the mean and multiplied by 100 . Statistical analyses were performed with a significance level of $5 \%$ $(\mathrm{p}<0.05)$. The statistical analysis was carried out with STATISTICA 12.5 software (StatSoft Inc., Tulsa, OK, USA).

\section{Results}

\section{Comparison of patients with naive acromegaly and the control group}

There were no significant differences in sex distribution, age, or BMI between patients and the control group. There were significantly more patients with hyperten- sion and diabetes in the acromegaly group than in the control group. A detailed comparison between patients and the control group in the selected variables is presented in Table 1. Echocardiographic findings are presented in Table 2. All measured parameters were significantly different between patients with naive acromegaly and the control group. The median LVMI differed significantly between groups. In the group of patients, there was a statistically significant negative correlation between LVMI and GLS $(\mathrm{R}=-0.47, \mathrm{p}<0.01)$ (Fig. 1).

Multivariate logistic regression analysis revealed that, of the selected variables, there were no significant independent predictive factors for abnormal GLS value (Tab. 3).

\section{Comparison between patients with normal and abnormal GLS values}

The majority of acromegalic patients ( $\mathrm{n}=26 ; 60.5 \%)$ had abnormal GLS values. Patients with impairment in GLS had a significantly longer median duration of acromegaly symptoms compared to those with normal GLS values $(\mathrm{p}<0.05)$. Patients with acromegaly and abnormal GLS had higher IGF-1 concentrations compared to those with normal GLS, although this parameter did not reach statistical significance. Acromegalics with abnormal GLS presented significantly higher values in one parameter of LV thickness, i.e. PW $(p<0.05)$. We did not find a difference in the prevalence of hypertension or glucose metabolism abnormalities in patients with normal vs. abnormal GLS. Detailed data are presented in Table 4. An example of GLS impairment $(-16.2 \%)$ in acromegaly patients is shown in Figure 2.

\section{Discussion}

Patients with newly diagnosed acromegaly presented abnormal GLS values, which allows us to draw con-

Table 1. Characteristics of patients and the control group regarding the occurrence of co-morbidities and biochemical parameters

\begin{tabular}{lccc}
\hline & Patients with acromegaly $(\mathbf{n}=\mathbf{4 3})$ & Control group $(\mathbf{n}=\mathbf{5 2})$ & $\mathbf{p}$ value \\
\hline Age $[$ years] & $48.2 \pm 14.2$ & $47.4 \pm 12.3$ & 0.76 \\
\hline Sex $($ female/male, $\mathrm{n})$ & $25 / 18$ & $27 / 25$ & 0.55 \\
\hline $\mathrm{BMI}\left[\mathrm{kg} / \mathrm{m}^{2}\right]$ & $29.8 \pm 5.0$ & $27.9 \pm 4.6$ & 0.06 \\
\hline BSA $\left[\mathrm{m}^{2}\right]$ & $2.01 \pm 0.23$ & $1.97 \pm 0.22$ & 0.33 \\
\hline Duration of acromegaly symptoms [years] & $10.0(2.0-40.0)$ & $\mathrm{N} / \mathrm{A}$ & - \\
\hline GH levels $[\mu \mathrm{g} / \mathrm{L}]$ & $8.5(1.0-79.0)$ & $\mathrm{N} / \mathrm{A}$ & - \\
\hline IGF-1 level $[\mu \mathrm{g} / \mathrm{L}]$ & $783.6(321.0-1600.0)$ & $\mathrm{N} / \mathrm{A}$ & - \\
\hline Hypertension $(Y e s /$ No) & $26 / 17$ & $17 / 35$ & $<0.01$ \\
\hline Diabetes status (Diabetes/Prediabetes/No) & $8 / 23 / 12$ & $0 / 3 / 49$ & $<0.001$ \\
\hline
\end{tabular}

$\mathrm{BMI}$ — body mass index; BSA — body surface area; GH — growth hormone; IGF-1 —insulin-like growth factor-1; N/A — not applicable 
Table 2. Echocardiography parameters in patients with acromegaly versus control group

\begin{tabular}{lccc}
\hline & $\begin{array}{c}\text { Patients with acromegaly } \\
\mathbf{( n = 4 3 )}\end{array}$ & $\begin{array}{c}\text { Control group } \\
\text { (n = 52) }\end{array}$ & p value \\
\hline Cardiac chamber size & $49.0(24.0-63.0)$ & $47.0(39.0-58.0)$ & $<0.05$ \\
\hline LVEDd $[\mathrm{mm}]$ & $43.0(27.3-97.3)$ & $29.9(15.9-53.3)$ & $<0.001$ \\
\hline LAVI $\left[\mathrm{mL} / \mathrm{m}^{2}\right]$ & & & $<0.001$ \\
\hline LV mass & $12.0(8.0-19.0)$ & $10.0(7.0-15.0)$ & $<0.001$ \\
\hline IVS $[\mathrm{mm}]$ & $12.0(8.0-17.0)$ & $10.0(7.0-14.0)$ & $<0.001$ \\
\hline PW $[\mathrm{mm}]$ & $136.0(77.0-236.0)$ & $97.0(44.0-163.0)$ & $<0.001$ \\
\hline LVMi $\left[\mathrm{g} / \mathrm{m}^{2}\right]$ & & & $<0.05$ \\
\hline Diastolic function & $7.0(4.0-12.0)$ & $9.0(5.0-18.0)$ & $<0.05$ \\
\hline E'med. $[\mathrm{cm} / \mathrm{s}]$ & $9.0(5.0-24.0)$ & $8.0(5.0-13.0)$ & $1.2(0.5-2.3)$ \\
\hline E/E' & $0.9(0.5-1.8)$ & & $<0.001$ \\
\hline E/A & & $-20.7(-14.6--30.2)$ & \\
\hline Systolic function & $-16.6(-8.4--26.5)$ & & \\
\hline GLS $(\%)$ & & & \\
\hline
\end{tabular}

LVEDd — left ventricular end diastolic diameter; LAVI — left atrial volume index; LV — left ventricular; IVS — interventricular septum; PW — posterior wall;

$\mathrm{LVMi}$ - left ventricular mass index; E'med. — early diastolic velocity of the septal mitral annulus; E/E' — mitral E-wave velocity divided by mitral annular velocity; E/A — mitral E-wave velocity divided by mitral A-wave velocity; GLS — global longitudinal strain

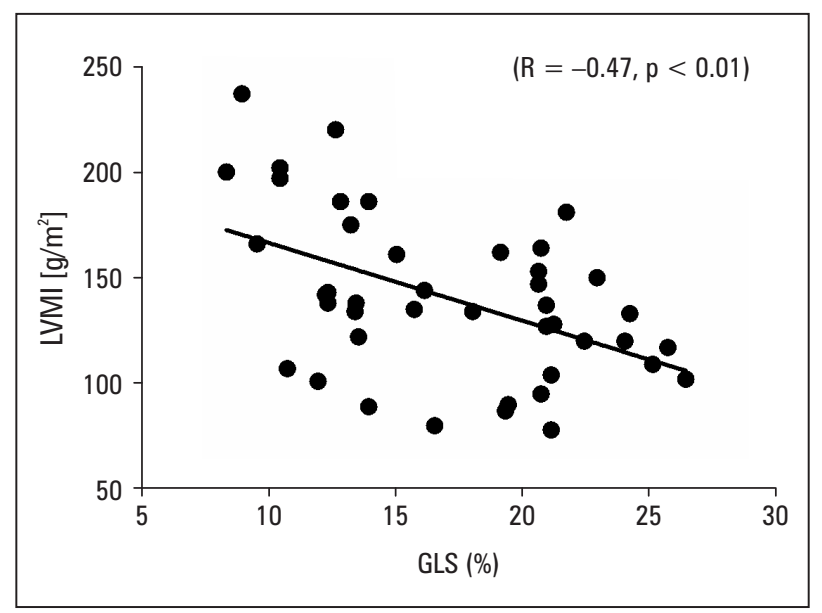

Figure 1. Correlation between left ventricular mass index (LVMI) and global longitudinal strain (GLS) in acromegaly group clusions about the occurrence of subclinical systolic dysfunction in this group. Only a few studies assessing deformation in patients with acromegaly have been published. Volschan et al. assessed GLS in 37 patients with active acromegaly and obtained normal values. Interestingly, these values were higher than in the control group. However, it should be noted that the examined group was heterogeneous, and most of the patients had already undergone surgery and were on somatostatin analogue treatment [9]. In our previous study, in which we performed echocardiography in 140 patients with acromegaly at different stages of treatment and disease activity, higher GLS values were obtained than in the group of untreated patients (GLS, in \%, -19.2 vs. -17.1) [20]. These findings may suggest a beneficial effect of treatment on the obtained GLS values. To eliminate the potential impact of the applied treatment on the

Table 3. Multivariate logistic regression analysis for global longitudinal strain (GLS)

\begin{tabular}{lcccc}
\hline Dependent variable & Predictors & Odds ratio & 95\% Cl & p value \\
\hline \multirow{4}{*}{ GLS } & Age & 0.97 & $0.90-1.05$ & 0.43 \\
\cline { 2 - 5 } & Sex & 0.85 & $0.21-3.49$ & 0.82 \\
\cline { 2 - 5 } & GH & 0.98 & $0.94-1.03$ & 0.48 \\
& IGF-1 & 1.00 & $0.99-1.01$ & 0.15 \\
\cline { 2 - 5 } & Hypertension & 0.56 & $0.09-3.59$ & 0.54 \\
\hline
\end{tabular}

GLS — global longitudinal strain (1 — abnormal value, 0 — value of normal range); $\mathrm{GH}$ — growth hormone; IGF-1 — insulin-like growth factor 1; $\mathrm{CI}$ — confidence interval 
Table 4. Characteristics of acromegalic patients with normal and abnormal global longitudinal strain (GLS)

\begin{tabular}{|c|c|c|c|}
\hline & $\begin{array}{l}\text { Patients with acromegaly } \\
\text { with normal GLS }\end{array}$ & $\begin{array}{l}\text { Patients with acromegaly } \\
\text { with abnormal GLS }\end{array}$ & $\mathrm{p}$ value \\
\hline Age [years] & $51.5 \pm 14.1$ & $46.0 \pm 14.1$ & 0.22 \\
\hline Sex (female/male) & $11 / 6$ & $14 / 12$ & 0.48 \\
\hline BMI $\left[\mathrm{kg} / \mathrm{m}^{2}\right]$ & $29.6 \pm 4.3$ & $29.9 \pm 5.5$ & 0.87 \\
\hline $\mathrm{BSA}\left[\mathrm{m}^{2}\right]$ & $2.01 \pm 0.22$ & $2.02 \pm 0.24$ & 0.88 \\
\hline Duration of acromegaly symptoms [years] & $5.0(2.0-40.0)$ & $10.0(3.0-40.0)$ & $<0.05$ \\
\hline $\mathrm{GH}[\mu \mathrm{g} / \mathrm{L}]$ & $5.7(1.0-79.2)$ & $11.2(2.2-70.1)$ & 0.18 \\
\hline IGF-1 [ $\mu \mathrm{g} / \mathrm{L}]$ & $673.0(408.0-1162.0)$ & $813.0(321.1-1600.0)$ & 0.07 \\
\hline Hypertension (Yes/No) & $10 / 7$ & $16 / 10$ & 0.86 \\
\hline Diabetes status (Diabetes/Prediabetes/No) & $3 / 10 / 4$ & $5 / 13 / 8$ & 0.84 \\
\hline \multicolumn{4}{|l|}{ Cardiac chamber size } \\
\hline LVEDd [mm] & $49.0(24.0-63.0)$ & $49.0(40.0-57.0)$ & 0.78 \\
\hline LAVI $\left[\mathrm{mL} / \mathrm{m}^{2}\right]$ & $39.9(27.3-61.9)$ & $46.2(29.6-97.3)$ & 0.13 \\
\hline \multicolumn{4}{|l|}{ LV mass } \\
\hline IVS [mm] & $12.0(8.0-14.0)$ & $12.5(8.0-19.0)$ & 0.08 \\
\hline PW [mm] & $12.0(9.0-13.0)$ & $12.5(8.0-17.0)$ & $<0.05$ \\
\hline LVMI [g/m²] & $126.0(77.0-180.0)$ & $141.5(79.0-236.0)$ & 0.08 \\
\hline \multicolumn{4}{|l|}{ Diastolic function } \\
\hline$E^{\prime}$ med. $[\mathrm{cm} / \mathrm{s}]$ & $8.0(4.0-11.0)$ & $6.5(4.0-12.0)$ & 0.54 \\
\hline $\mathrm{E} / \mathrm{E}^{\prime}$ & $9.0(6.0-15.0)$ & $9.0(5.0-24.0)$ & 0.95 \\
\hline$E / A$ & $0.9(0.6-1.5)$ & $0.9(0.5-1.8)$ & 0.99 \\
\hline
\end{tabular}

BMI — body mass index; BSA — body surface area; GH — growth hormone; IGF-1 —insulin-like growth factor-1; LVEDd — left ventricular end diastolic diameter; LAVI — left atrial volume index; LV — left ventricular; IVS — interventricular septum; PW — posterior wall; LVMi — left ventricular mass index; E'med. — early diastolic velocity of the septal mitral annulus; E/E' - mitral E-wave velocity divided by mitral annular velocity; E/A — mitral E-wave velocity divided by mitral A-wave velocity; GLS - global longitudinal strain
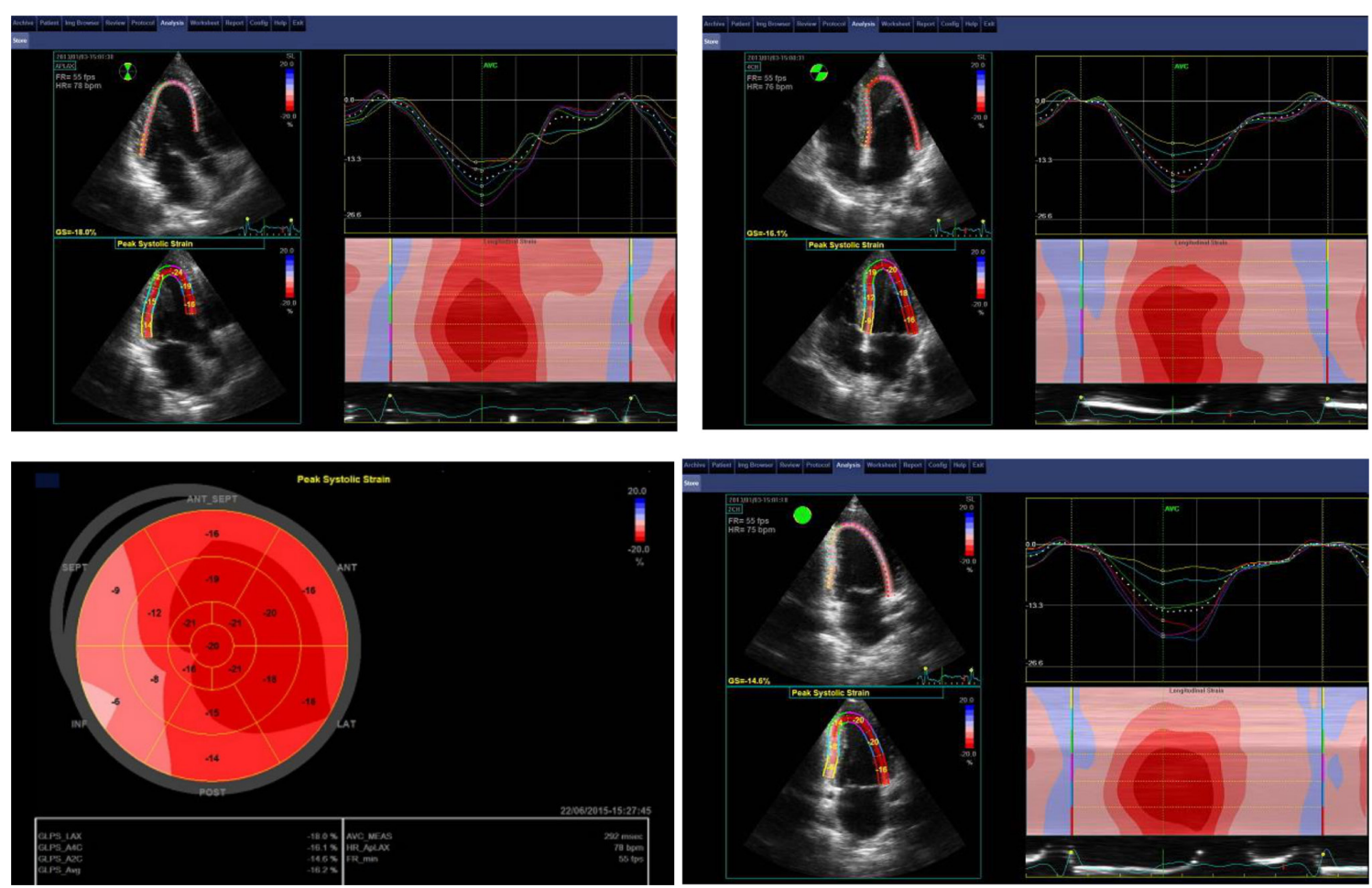

Figure 2. Global longitudinal strain (GLS) impairment in patients with naive acromegaly 
results, we studied a homogeneous group of patients with acromegaly before treatment. To the best of our knowledge, this is the first study that has evaluated GLS with the speckle-tracking technique in a group of naive acromegalic patients.

The results of our study demonstrate that naive acromegaly patients had larger LVMI than the control subjects, and there was a negative correlation between LVMI and GLS.

To date, many studies have assessed GLS in diseases accompanied by left ventricle hypertrophy, such as hypertension, diabetes mellitus, and Cushing's disease $[7,21,22]$.

The impact of traditional cardiovascular disease risk factors, such as arterial hypertension, diabetes mellitus, lipid disorders, and insulin resistance, on the microvascular system is well established [23]. It involves endothelial and smooth muscle cell dysfunction and vascular remodelling [23-26].

Because of this pathophysiology, abnormal GLS in this group of diseases is expected. It is interesting that hypertension and diabetes mellitus, which are significantly more common in the presence of acromegaly, were not significantly associated with GLS value in our study group. Similarly, other parameters, such as age, sex, BMI, and GH and IGF-1 concentrations, were not significantly different between the group with normal GLS results and the group with abnormal GLS values. IGF-1 was the most influential factor among these, but it did not reach statistical significance.

The activity of the IGF-1/2 system on endothelial cells is multidirectional, both protective and pathologi$\mathrm{cal}$, in a range of diseases. Hence, there are objective difficulties in interpreting the effects of elevated IGF-1 in acromegalic patients. Both of these proteins play important roles in maintaining homeostasis by endothelial cells [27-29]. Insulin-like growth factor-1 regulates vascular tone by decreasing the response to vasoconstrictor hormones, such as angiotensin II, norepinephrine and vasopressin, and through vascular production of the vasodilator nitric oxide $(\mathrm{NO})$ [30].

Some studies suggest that IGF-1 promotes vascular smooth muscle cell proliferation and migration and that its expression is increased in atherosclerotic plaques, and in this sense, it is proatherogenic $[27,31]$. On the other hand, IGF-1 is also protective both by enhancing endothelial cell function and by promoting plaque stability through increased vascular smooth muscle cell survival $[27,28]$.

Low IGF-1 levels result in insulin resistance and accelerated atherosclerosis and consequently correlate with an increased risk of adverse cardiovascular outcomes, including myocardial infarction and heart failure [27, 32]. Additionally, IGF-1 affects the number and function of endothelial progenitor cells, which decrease with age. These adverse changes can be reversed by increasing IGF-1 levels with GH treatment [27, 33].

In a study by Tellatin et al. the relationship of coronary microvascular impairment with elevated levels of IGF-1 in acromegalic patients was assessed [34]. Forty patients (28 with active acromegaly) were included in the study, and all had non-invasive coronary flow reserve (CFR) assessment using transthoracic doppler echocardiography. Thirteen patients had abnormal results (CFR < 2.5), which independently correlated with IGF-1 level. In addition, CFR was improved during treatment in some patients.

Anagnostis et al. also showed an adverse effect of active acromegaly on endothelial function [35]. They evaluated total antioxidant capacity measured by catalase activity and glutathione concentration, oxidative stress measured by oxidised glutathione and thiobarbituric acid-reactive substances, and NO levels. They found increased oxidative stress, decreased antioxidative capacity, and reduced NO levels in acromegalic patients compared to controls.

Our findings are similar and show that patients with active untreated acromegaly with normal EF have subclinical systolic dysfunction as assessed by decreased GLS, which is probably due to the negative effect of IGF-1 on the microvascular system. In our group of patients with normal GLS (39.5\%), the mean IGF-1 value was only nominally lower than in the group with abnormal GLS $(741.9 \pm 245.1$ vs. $932.3 \pm 350.8)$; the difference was not statistically significant, although this value was clearly above the norm for the patients' age and sex. The occurrence of hypertension and diabetes did not differ significantly between the groups. It can be argued that the reason we obtained good GLS results is that the positive effect of IGF-1 predominates over its pathological effect on the microvascular system.

However, there is an open and interesting question about why other patients with high IGF-1 values have abnormal GLS. Perhaps there is an IGF-1 value above which its detrimental effect on endothelial cells dominates. Maybe patients treated with somatostatin analogues do not have to reach the norm for age and sex, and maybe the group of patients with no improvement in GLS values should be treated for prevention of overt cardiac failure. This is an interesting issue for further studies.

\section{Study limitations}

The study was limited by the fact that until 2012, examinations were performed using the Vivid 4 echocardiograph, which had lower technical capabilities than the Vivid 9 device. The Vivid 9 device was used for the majority of patients and all controls. Because GLS is 
partially dependent on the echocardiograph and the applied software as well as the researcher's experience, comparing the results obtained in our study with the results of other studies can be a limitation. Data regarding the duration of symptoms were self-reported by patients, which means the data are not accurate and constitute a limitation of this study. Another study limitation was the low number of patients.

\section{Conclusions}

Naive acromegalic patients presented with lower GLS compared to the control group, revealing subclinical systolic dysfunction in patients with naive, untreated acromegaly.

It has not been proven that arterial hypertension and diabetes mellitus, which are significantly more common in acromegaly, are significant determinants of abnormal GLS.

\section{Authors' contributions}

Conceived and designed the experiment: A.P.G., M.S.B., M.D., W.Z. The acquisition, analysis, or interpretation of data for the work: A.P.G., JSG, M.C., P.G. Drafting the work or revising it critically for important intellectual content: A.P.G., M.S.B., J.S.G., P.G. Final approval of the version to be published: A.P.G., M.S.B., J.S.G., P.G., M.C., M.D., W.Z., W.K. Agreement to be accountable for all aspects of the work in ensuring that questions related to the accuracy or integrity of any part of the work are appropriately investigated and resolved: A.P.G., M.S.B., J.S.G., M.D., W.Z., W.K.

\section{Conflict of interest statement}

The authors declare no conflict of interest.

\section{Data availability statement}

Derived data supporting the findings of this study are available from the corresponding author [mstelmachowska@cmkp.edu.pl] on request.

\section{References}

1. Bolanowski M, Ruchała M, Zgliczyński W, et al. Diagnostics and treatment of acromegaly - updated recommendations of the Polish Society of Endocrinology. Endokrynol Pol. 2019; 70(1): 2-18, doi: 10.5603/EP.a2018.0093, indexed in Pubmed: 30843181.

2. Stelmachowska-Banaś M, Głogowski M, Vasiljevic A, et al. Ectopic acromegaly due to growth hormone-releasing hormone secretion from bronchial carcinoid causing somatotroph hyperplasia and partial pituitary insufficiency. Pol Arch Intern Med. 2019; 129(3): 208-210, doi: 10.20452/pamw.4413, indexed in Pubmed: 30608060.

3. Vilar L, Vilar CF, Lyra R, et al. Acromegaly: clinical features at diagnosis. Pituitary. 2017; 20(1): 22-32, doi: 10.1007/s11102-016-0772-8, indexed in Pubmed: 27812777.

4. Ponikowski P, Voors AA, Anker SD, et al. ESC Scientific Document Group. 2016 ESC Guidelines for the diagnosis and treatment of acute and chronic heart failure: The Task Force for the diagnosis and treatment of acute and chronic heart failure of the European Society of Cardiology (ESC) Developed with the special contribution of the Heart Failure Association
(HFA) of the ESC. Eur Heart J. 2016; 37(27): 2129-2200, doi: 10.1093/eurheartj/ehw128, indexed in Pubmed: 27206819

5. Tops LF, Delgado V, Marsan NA, et al. Myocardial strain to detect subtle left ventricular systolic dysfunction. Eur J Heart Fail. 2017; 19(3): 307-313, doi: 10.1002/ejhf.694, indexed in Pubmed: 27891719.

6. Varghese MJ, Sharma G, Shukla G, et al. Longitudinal ventricular systolic dysfunction in patients with very severe obstructive sleep apnea: A case control study using speckle tracking imaging. Indian Heart J. 2017; 69(3): 305-310, doi: 10.1016/j.ihj.2016.12.011, indexed in Pubmed: 28648418.

7. Uziębło-Życzkowska B, Krzesinński P, Witek P, et al. Cushing's Disease: Subclinical Left Ventricular Systolic and Diastolic Dysfunction Revealed by Speckle Tracking Echocardiography and Tissue Doppler Imaging. Front Endocrinol (Lausanne). 2017; 8: 222, doi: 10.3389/fendo.2017.00222, indexed in Pubmed: 28928716

8. Geyer H, Caracciolo G, Abe H, et al. Assessment of myocardial mechanics using speckle tracking echocardiography: fundamentals and clinical applications. J Am Soc Echocardiogr. 2010; 23(4): 351-69; quiz 453, doi: 10.1016/j.echo.2010.02.015, indexed in Pubmed: 20362924.

9. Volschan ICM, Kasuki L, Silva CMS, et al. Two-dimensional speckle tracking echocardiography demonstrates no effect of active acromegaly on left ventricular strain. Pituitary. 2017; 20(3): 349-357, doi: 10.1007/s11102-017-0795-9, indexed in Pubmed: 28220351.

10. Lang RM, Badano LP, Mor-Avi V, et al. Recommendations for cardiac chamber quantification by echocardiography in adults: an update from the American Society of Echocardiography and the European Association of Cardiovascular Imaging. J Am Soc Echocardiogr. 2015; 28(1): 1-39.e14, doi: 10.1016/j.echo.2014.10.003, indexed in Pubmed: 25559473.

11. Gunasekaran P, Panaich S, Briasoulis A, et al. Incremental Value of Two Dimensional Speckle Tracking Echocardiography in the Functional Assessment and Characterization of Subclinical Left Ventricular Dysfunction. Curr Cardiol Rev. 2017; 13(1): 32-40, doi: 10.2174/1573403x126661 60712095938, indexed in Pubmed: 27411342.

12. Stampehl MR, Mann DL, Nguyen JS, et al. Speckle strain echocardiography predicts outcome in patients with heart failure with both depressed and preserved left ventricular ejection fraction. Echocardiography. 2015 32(1): 71-78, doi: 10.1111/echo.12613, indexed in Pubmed: 24816065.

13. Smiseth OA, Torp H, Opdahl A, et al. Myocardial strain imaging: how useful is it in clinical decision making? Eur Heart J. 2016; 37(15): 1196-1207, doi: 10.1093/eurheartj/ehv529, indexed in Pubmed: 26508168

14. Tykarski A, Narkiewicz K, Gaciong Z, et al. 2015 guidelines for the management of hypertension. Recommendations of the Polish Society of Hypertension - short version. Kardiol Pol. 2015; 73(8): 676-700, doi: 10.5603/KP.2015.0157, indexed in Pubmed: 26304155

15. Araszkiewicz A, et al. Bandurska-Stankiewicz, E, Budzyński A, A position of Diabetes Poland. 2018 Guidelines on the management of diabetic patients. Clin Diabetol. 2018; 7: 1-90, doi: 10.5603/DK.2019.0001.

16. Belghitia H, Brette $S$, Lafitte $S$, et al. Automated function imaging: a new operator-independent strain method for assessing left ventricular function. Arch Cardiovasc Dis. 2008; 101(3): 163-169, doi: 10.1016/s1875-2136(08)71798-4, indexed in Pubmed: 18477943.

17. Devereux RB, Alonso DR, Lutas EM, et al. Echocardiographic assessment of left ventricular hypertrophy: comparison to necropsy findings. Am J Cardiol. 1986; 57(6): 450-458, doi: 10.1016/0002-9149(86)90771-x, indexed in Pubmed: 2936235.

18. Nagueh SF, Smiseth OA, Appleton CP, et al. Recommendations for the Evaluation of Left Ventricular Diastolic Function by Echocardiography: An Update from the American Society of Echocardiography and the European Association of Cardiovascular Imaging. J Am Soc Echocardiogr. 2016; 29(4): 277-314, doi: 10.1016/j.echo.2016.01.011, indexed in Pubmed: 27037982.

19. Cole GD, Dhutia NM, Shun-Shin MJ, et al. Defining the real-world reproducibility of visual grading of left ventricular function and visual estimation of left ventricular ejection fraction: impact of image quality, experience and accreditation. Int J Cardiovasc Imaging. 2015; 31(7) 1303-1314, doi: 10.1007/s10554-015-0659-1, indexed in Pubmed: 26141526.

20. Popielarz-Grygalewicz A, Gąsior JS, Konwicka A, et al. Heart in Acromegaly: The Echocardiographic Characteristics of Patients Diagnosed with Acromegaly in Various Stages of the Disease. Int J Endocrinol. 2018. 2018: 6935054, doi: 10.1155/2018/6935054, indexed in Pubmed: 30123265 .

21. Fung MJ, Thomas L, Leung DY, et al. Left ventricular function and contractile reserve in patients with hypertension. Eur Heart J Cardiovasc Imaging. 2018; 19(11): 1253-1259, doi: 10.1093/ehjci/jex338, indexed in Pubmed: 29300855.

22. Wang $\mathrm{Q}$, Tan $\mathrm{K}$, Xia $\mathrm{H}$, et al. Left ventricular structural alterations are accompanied by subclinical systolic dysfunction in type 2 diabetes mellitus patients with concomitant hyperlipidemia: An analysis based on 3D speckle tracking echocardiography. Echocardiography. 2018; 35(7): 965-974, doi: 10.1111/echo.13858, indexed in Pubmed: 29509974.

23. Crea F, Camici PG, Bairey Merz CN. Coronary microvascular dysfunction: an update. Eur Heart J. 2014; 35(17): 1101-1111, doi: 10.1093/eurheartj/eht513, indexed in Pubmed: 24366916. 
24. Antony I, Nitenberg A, Foult JM, et al. Coronary vasodilator reserve in untreated and treated hypertensove patients with and without left ventricular hypertrophy. J Am Coll Cardiol. 1993; 22(2): 514-520, doi: 10.1016/0735-1097(93)90058-9, indexed in Pubmed: 8335823

25. Rizzoni D, Palombo C, Porteri E, et al. Relationships between coronary flow vasodilator capacity and small artery remodelling in hypertensive patients. J Hypertens. 2003; 21(3): 625-631, doi: 10.1097/00004872-20030 3000-00030, indexed in Pubmed: 12640258.

26. Nitenberg A, Valensi P, Sachs R, et al. Impairment of coronary vascular reserve and ACh-induced coronary vasodilation in diabetic patients with angiographically normal coronary arteries and normal left ventricular systolic function. Diabetes. 1993; 42(7): 1017-1025, doi: 10.2337/diab.42.7.1017, indexed in Pubmed: 8513969

27. Bach LA. Endothelial cells and the IGF system. J Mol Endocrinol. 2015; 54(1): R1-13, doi: 10.1530/JME-14-0215, indexed in Pubmed: 25351818.

28. Holly JMP, Perks CM. Insulin-like growth factor physiology: what we have learned from human studies. Endocrinol Metab Clin North Am. 2012; 41(2): 249-63, v, doi: 10.1016/j.ecl.2012.04.009, indexed in Pubmed: 22682629.

29. Filus A, Zdrojewicz Z. [Insulin-like growth factor-1 (IGF-1) - structure and the role in the human body]. Pediatr Endocrinol Diabetes Metab. 2015; 20(4): 161-169, doi: 10.18544/PEDM-20.04.0016, indexed in Pubmed: 26615583
30. Muniyappa R, Walsh MF, Rangi JS, et al. Insulin like growth factor 1 increases vascular smooth muscle nitric oxide production. Life Sci. 1997; 61(9): 925-931, doi: 10.1016/s0024-3205(97)00594-8, indexed in Pubmed: 9284085.

31. Clemmons DR. Modifying IGF1 activity: an approach to treat endocrine disorders, atherosclerosis and cancer. Nat Rev Drug Discov. 2007; 6(10): 821-833, doi: 10.1038/nrd2359, indexed in Pubmed: 17906644.

32. Higashi Y, Sukhanov S, Anwar A, et al. Aging, atherosclerosis, and IGF-1. J Gerontol A Biol Sci Med Sci. 2012; 67(6): 626-639, doi: 10.1093/gerona/gls102, indexed in Pubmed: 22491965.

33. Thum T, Hoeber S, Froese S, et al. Age-dependent impairment of endothelial progenitor cells is corrected by growth-hormone-mediated increase of insulin-like growth-factor-1. Circ Res. 2007; 100(3): 434-443, doi: 10.1161/01.RES.0000257912.78915.af, indexed in Pubmed: 17234973.

34. Tellatin S, Maffei P, Osto E, et al. Coronary microvascular dysfunction may be related to IGF-1 in acromegalic patients and can be restored by therapy. Atherosclerosis. 2018; 269: 100-105, doi: 10.1016/j.atherosclerosis.2017.12.019, indexed in Pubmed: 29353224.

35. Anagnostis P, Efstathiadou ZA, Gougoura S, et al. Oxidative stress and reduced antioxidative status, along with endothelial dysfunction in acromegaly. Horm Metab Res. 2013; 45(4): 314-318, doi: 10.1055/s-0032-1323765, indexed in Pubmed: 23093460. 